\title{
THE INDICES OF TORSION-FREE SUBGROUPS OF FUCHSIAN GROUPS
}

\author{
R. G. BURNS AND DONALD SOLITAR ${ }^{1}$
}

\begin{abstract}
Elementary algebraic techniques are used to obtain the precise possible indices of torsion-free subgroups of finite index of finitely generated Fuchsian groups (and related groups).
\end{abstract}

Introduction. In a recent paper, Edmonds, Ewing and Kulkarni [3] announced the precise finite indices possible for a torsion-free subgroup of a finitely generated Fuchsian group (of orientation-preserving isometries of the hyperbolic plane). The proof (as described by the authors) is of a geometric-topological nature (they state that it contains "an involved sequence of explicit constructions of tesselations coupled with inductions and branched covering arguments"). In the present paper we give an elementary algebraic proof based on the permutation representations of the triangle groups, given by Fox [4] in his proof of Fenchel's conjecture, that every finitely generated Fuchsian group has a torsion-free subgroup of finite index. (Note that Fox's paper contains an error, corrected by Chau [1].)

Preliminaries. The finitely generated Fuchsian groups are characterized algebraically by the presentation

$$
\begin{aligned}
& \left\langle e_{1}, \ldots, e_{r}, a_{1}, b_{1}, \ldots, a_{g}, b_{g}, y_{1}, \ldots, y_{s} ;\right. \\
& \left.\quad e_{1}^{m_{1}}, \ldots, e_{r}^{m_{r}},\left[a_{1}, b_{1}\right] \cdots\left[a_{g}, b_{g}\right] e_{1} \cdots e_{r} y_{1} \cdots y_{s}\right\rangle,
\end{aligned}
$$

where $r, g, s \geqslant 0$ but not all $0, m_{i}>1$ for $i=1, \ldots, r$, and $[a, b]$ denotes the commutator $a b a^{-1} b^{-1}$. The finitely generated non-orientation-preserving subgroups of the hyperbolic plane which contain no reflections are characterized algebraically by the presentation

$$
\left\langle e_{1}, \ldots, e_{r}, a_{1}, \ldots, a_{g}, y_{1}, \ldots, y_{s} ; e_{1}^{m_{1}}, \ldots, e_{r}^{m_{r}}, a_{1}^{2} \cdots a_{g}^{2} e_{1} \cdots e_{r} y_{1} \cdots y_{s}\right\rangle,
$$

where $r, g, s \geqslant 0$ but not all $0, m_{i}>1$ for $i=1, \ldots, r$.

TheOREM 1 (EDMONDS, EwING AND KULKARNi). Let $c=\operatorname{lcm}\left(m_{1}, \ldots, m_{r}\right)$ be the least common multiple of the exponents on $e_{1}, \ldots, e_{r}$ in (1) (or in (2)) [note: if $r=0$ then $c=1]$, and let $d$ be the remainder $\bmod 2$ of $2^{s}$ times the number of even $m_{1}, \ldots, m_{r}$

\footnotetext{
Received by the editors August 1, 1982 and, in revised form, February 3, 1983. 1980 Mathematics Subject Classification. Primary 20H10; Secondary 20 F05.

Key words and phrases. Fuchsian group, index of a subgroup, torsion-free group.

${ }^{1}$ This research was supported by NSERC grants A7839 and A5614. $0002-9939 / 83 \$ 1.00+\$ .25$ per page
} 
having the same exponent on 2 in their prime factorization as $c$. A torsion-free subgroup of finite index $k$ exists in an infinite group with presentation (1) (or (2)) iff $k$ is divisible by $2^{d} c$.

In our proof of this theorem, the following two well-known results will be used repeatedly.

LEMMA 1. A subgroup of finite index in a group with presentation (1) or (2) also has such a presentation.

LEMMA 2. Any element of finite order in an infinite group with presentation (1) or (2) is conjugate to a power of some $e_{i}$. Moreover, the order of $e_{i}$ is $m_{i}$.

For algebraic proofs of these lemmas see Hoare, Karrass and Solitar [5 and 6].

We shall also use the following result of Fox [4, cases II and III], which has an elementary algebraic proof (corrected in Chau [1]).

LEMma 3. If $k, m$ and $n$ are three positive integers with $k \geqslant m, n$, such that mnk is odd, or else $m n$ is even and $k \geqslant m+n$, then there exist permutations $\alpha$ and $\omega$ in $S_{k}$ (the symmetric group on $k$ symbols) of orders $m, n$ respectively such that $\alpha \omega=(1$ $\cdots k)$.

We can now give a proof of the theorem. Throughout $G$ will denote an infinite group with presentation (1) or (2) unless otherwise indicated. The following lemma establishes the necessity of the condition on the indices of torsion-free subgroups of finite index.

LEMMA 4. A torsion-free subgroup of finite index in an infinite group with presentation (1) or (2) must have index divisible by c. Moreover, if $d=1$ then the index must be divisible by $2 c$.

Proof. If $H$ is a torsion-free subgroup of $G$ and $J$ is a finite subgroup, then obviously $J$ has trivial intersection with any conjugate of $H$. Hence each $(J, H)$-double-coset of $G$ has $|J| H$-cosets in it, and therefore $[G: H]$ is divisible by $|J|$. Thus $c \mid[G: H]$. Moreover, each element of $J$ defines a regular (i.e., fixed-point-free) permutation of the $H$-cosets. Hence, if $\left[G: H\right.$ ] is an odd multiple of $c$, then each $e_{i}$ with even order $m_{i}$ having the same 2-exponent as $c$, defines a permutation with an odd number of even cycles, i.e., an odd permutation, while the other $e_{i}$ define even permutations. But the product relation in (1) or (2) must define an even permutation (since it defines the identity), and moreover, a commutator or a square defines an even permutation. Hence, if $s=0$, the product of the permutations corresponding to the $e_{i}$ must be even. Thus, if $d=1$ we get a contradiction of our assumption that $[G: H]$ is an odd multiple of $c$.

Sufficiency. An infinite torsion-free group with presen:ation (1) or (2) has $r=0$, and clearly has an infinite cyclic quotient group and, therefore, torsion-free subgroups of any finite index. Hence, to prove the theorem, it suffices to show that any group $G$ with presentation (1) or (2) has a torsion-free subgroup of index $c$ if $d=0$, and of index $2 c$ if $d=1$. 
Certain cases on the values of $r, s$ and $g$ are relatively easy, and we first dispose of these.

When $r=0, G$ itself is torsion-free and $c=1, d=0$, and so this case is clear. When $s \geqslant 1, G$ is a free product of cyclic groups, and may be mapped onto a cyclic group of order $c$, with $e_{i}$ being mapped into the $\left(c / m_{i}\right)$ th power of the generator while the other generators are mapped into 1 , and so this case is also clear. Thus we may assume from now on that $s=0$ and $r \geqslant 1$.

If $r=1$ then, since $G$ is infinite, $g \geqslant 1$. For (1) we can map $G$ into $D_{2 c}=$ $\left\langle a, b ; a^{2 c}, b^{2},(a b)^{2}\right\rangle$, the dihedral group of order $4 c$, with $e_{1} \rightarrow a^{-4}, a_{1} \rightarrow a^{2}, b_{1} \rightarrow b$ if $c$ is odd, and $e_{1} \rightarrow a^{-2}, a_{1} \rightarrow a, b_{1} \rightarrow b$ if $c$ is even; the other generators are mapped into 1. The preimage in $G$ of $\operatorname{gp}(b)$ under the described map will be torsion-free and have the required index. For (2) we can map $G$ onto $Z_{c}=\left\langle a ; a^{c}\right\rangle$, the cyclic group of order $c$, with $e_{1} \rightarrow a^{-2}, a_{1} \rightarrow a$, if $c$ is odd, and map $G$ onto $Z_{2 c}=\left\langle a ; a^{2 c}\right\rangle$, the cyclic group of order $2 c$, with $e_{1} \rightarrow a^{-2}, a_{1} \rightarrow a$, if $c$ is even; the other generators are mapped into 1 . The kernel of this map will be torsion-free and have the required index.

If $r=2$ then, again, $g \geqslant 1$. If $d=1$ or $c$ is odd we reduce this case to a previous one by mapping $G$ onto the group with presentation (1) or (2) and $r=1, m_{1}=c$, in effect by mapping the cyclic groups generated by $e_{1}$ and $e_{2}$ into their amalgamated direct product, a cyclic group of order $c$ generated by $e_{1} e_{2}$, and leaving the other generators as they are. If $c$ is even and $d=0$, then $m_{1}$ and $m_{2}$ both have the same highest power of two in them, say $2^{p}$. Let $m_{i}=2^{p} n_{i}$ for $i=1,2$, and let the cyclic group $\operatorname{gp}(a)$ of order $2 c$ be the direct product of the cyclic group $\operatorname{gp}(u)$ of order $2^{p+1}$ and the cyclic group $\operatorname{gp}(v)$ of odd order $q$. If $G$ has presentation (1), then we map $G$ into the dihedral group $D_{2 c}$ by sending $e_{1} \rightarrow u^{2} v^{t}$ and $e_{2} \rightarrow u^{2} v^{x}$, where $t=q / n_{1}$ and $x=q / n_{2}$. Then $e_{1} e_{2}$ is mapped onto a fourth power of some element $z$ in $\operatorname{gp}(a)$. Hence, if $a_{1} \rightarrow z^{-2}, b_{1} \rightarrow b$, and the other generators are sent into 1 , the image of $G$ will be $\operatorname{gp}\left(a^{2}, b\right)$ and the preimage of $\operatorname{gp}(b)$ will be torsion-free with index $c$. If $G$ has presentation (2) we map $G$ into $D_{2 c}$ as above (but of course there is no $b_{1}$ ). The kernel of this mapping will be torsion-free with index $c$.

If $g \geqslant 1$, then by mapping all $a_{i}, b_{i}$ into 1 and leaving the $e_{i}$ 's as they are, we clearly reduce the problem to that of finding torsion-free subgroups of the appropriate index in the finite and infinite groups having presentation (1) with $r \geqslant 3$ and $g=s=0$, i.e.,

$$
G=\left\langle e_{1}, \ldots, e_{r} ; e_{1}^{m_{1}}, \ldots, e_{r}^{m_{r}}, e_{1} \cdots e_{r}\right\rangle, \quad r \geqslant 3 .
$$

This group will be finite iff $r=3$ and $\left(m_{1}, m_{2}, m_{3}\right)$ is, in some order, one of the triples $(2,2, n),(2,3,3),(2,3,4)$ or $(2,3,5)$. In each of these cases, the trivial subgroup 1 is torsion-free and has the appropriate index (which is the order of $G$ ) (see Coxeter and Moser [2]). We may therefore assume that group $G$ in (3) is infinite.

We shall use induction on $c$.

Case 1. $d=0, c$ is odd, and the $m_{i}$ are coprime in pairs.

Let $p, p_{2}, p_{3}$ be three distinct (odd) primes dividing three of the $m_{i}$, say, $m_{1}, m_{2}$, $m_{3}$, respectively, with $p \geqslant p_{2}, p_{3}$. By Lemma 3 there exist permutations $\alpha$ of order $p_{2}$ 
and $\omega$ of order $p_{3}$ in $S_{p}$, the symmetric group on $p$ objects, such that $(1 \cdots p)=\alpha \omega$. The mapping $e_{1} \rightarrow(1 \cdots p), e_{2} \rightarrow \omega^{-1}, e_{3} \rightarrow \alpha^{-1}$, and $e_{i} \rightarrow 1$ for $i>3$, defines a homomorphism $\rho$ from $G$ into $S_{p}$. Since the image $\rho G$ is transitive in $S_{p}$, the stabilizer of 1 in $\rho G$ has index $p$ in $\rho G$; hence, if $H$ is the preimage of the stabilizer of 1 under $\rho$ in $G$ then $[G: H]=p$. Clearly, from Lemma 2 , the orders of the elements of $H$ divide those of $G$; moreover, some power of $e_{1}$ is in $H$ iff $p$ divides that power; also, some conjugate of $e_{2}$ and of $e_{3}$ is in $H$. It then follows from Lemmas 1 and 2 that $H$ has presentation (1) or (2) with the lcm of the powers of its $e_{i}$ equal to $c / p$. The torsion-free subgroup of $H$ of index $c / p$, which exists by inductive hypothesis, will then have the required index $c$ in $G$.

Case 2. $d=0, c$ is odd, the $m_{i}$ are not coprime in pairs.

Let $p$ be an odd prime which divides at least two of the $m_{i}$ 's, say $m_{1}, \ldots, m_{k}$, where $k \geqslant 2$, and no other $m_{i}$. Let $f_{i}=0$ if $i>k, f_{i}=1$ if $3 \leqslant i \leqslant k$, while $f_{1}=1$ and $f_{2}=p-1$ if $p$ divides $k-2$, but $f_{1}=f_{2}=(2-k) / 2 \bmod p$ if $p$ does not divide $k-2$. Clearly the mapping $e_{i} \rightarrow f_{i}$ defines a homomorphism $\rho$ of $G$ onto $Z_{p}$, the cyclic group of integers $\bmod p$. Some power of $e_{i}$ for $1 \leqslant i \leqslant k$ is in $H$, the kernel of $\rho$, iff $p$ divides that power. Hence, by Lemmas 1 and $2, H$ has a presentation (1) or (2) with the lcm of its $m_{i}$ equal to $c / p$. The subgroup of $H$ given by the inductive hypothesis is torsion-free with index $c$ in $G$.

Case 3. $d=0$ and $c$ is even.

Suppose $c=2^{p} q$ where $q$ is odd. Since $d=0$ and $c$ is even, $p>0$ and there are an even number of $m_{i}$ 's such that $2^{p}$ divides $m_{i}$, say $1 \leqslant i \leqslant k$, where $k$ is even. Define $p(i)$ to be the highest power of 2 in $m_{i}$. We define a homomorphism $\rho$ of $G$ onto $Z_{2^{k}}$, the cyclic group of order $2^{p}$, by mapping $e_{i}$ to $2^{p} / 2^{p(i)}$ for $2 \leqslant i \leqslant r$, and mapping $e_{1}$ to $f$ where $f$ is the odd number such that $f$ added to the sum of the images of the other $e_{i}$ is $0 \bmod 2^{p}$. (Since $k-1$ of the images of the other $e_{i}$ 's are 1 and the remaining images are even, it follows that $f$ is indeed odd.) Clearly a power of some $e_{i}$ is in $H$, the kernel of $\rho$, iff $2^{p(i)}$ divides that power; moreover, $[G: H]=2^{p}$. Hence, by Lemmas 1 and $2, H$ has only elements of odd order, and in the presentation (1) or (2) for $H$, the $\mathrm{lcm}$ of its $m_{i}$ is $q=c / 2^{p}$. The torsion-free subgroup of $H$ guaranteed by the inductive hypothesis then has index $c$ in $G$.

Case 4. $d=1$, at least 2 even $m_{i}$.

Suppose $m_{1}, m_{2}$ are even, where $m_{1}$ has the same 2-exponent as $c$. Consider the homomorphism $\rho$ of $G$ onto $Z_{2}$, the group of integers $\bmod 2$, given by $e_{1} \rightarrow 1$, $e_{2} \rightarrow 1$, and all other $e_{i} \rightarrow 0$. Clearly a power of $e_{1}$ or $e_{2}$ is in $H$, the kernel of $\rho$, iff the power is even; clearly [G:H] $=2$. If $m_{1}$ is the only $m_{i}$ with the same 2-exponent as $c$, then $H$ has a presentation (1) or (2) in which the lcm of its $m_{i}$ is $c / 2$. Now $H$ cannot have a torsion-free subgroup of index $c / 2$, since then $G$ would have a torsion-free subgroup of index $c$, contrary to Lemma 4. Hence, by inductive hypothesis $H$ has a torsion-free subgroup of index $c=2(c / 2)$, and this has index $2 c$ in $G$ as required.

On the other hand, since $d=1$, if some other $m_{i}$, in addition to $m_{1}$, has the same 2-exponent as $c$, then there are at least three such, so we may suppose the $1 \mathrm{~cm}$ of the presentation (1) or (2) for $H$ is still $c$. Using a Reidemeister-Schreier rewriting 
process to obtain a specific presentation for $H$ with $1, e_{1}$ as Schreier coset representative system (see Magnus, Karrass and Solitar [7]), and simplifying a bit, we obtain a presentation of type (1) with $g=s=0$ and generators $f_{1}=e_{1}^{2}, f_{2}=e_{2}^{2}$, $f_{3}=e_{3}, \ldots, f_{r}=e_{r}$, and $g_{3}=e_{1} e_{3} e_{1}^{-1}, \ldots, g_{r}=e_{1} e_{r} e_{1}^{-1}$. The exponents in the power relations are $m_{1} / 2, m_{2} / 2, m_{3}, \ldots, m_{r}, m_{3}, \ldots, m_{r}$. Hence, the number of generators with maximum 2-exponent is even, i.e. for $H, d=0$ and $c$ is even, Case 3 applies and therefore $H$ has a torsion-free subgroup of index $c$ which is of index $2 c$ in $G$ as required.

Case 5. $m_{1}$ even, $m_{i}$ odd for $i>1$, some pair of $m_{i}$ not coprime.

Let $p$ be an (odd) prime which divides at least two of the $m_{i}$ 's. Then we construct a homomorphism of $G$ into $Z_{p}$ as in Case 2, and a subgroup $H$ with $[G: H]=p$ and with a presentation (1) or (2) in which the $\mathrm{lcm}$ is $c / p$. Now by inductive hypothesis $H$ has a torsion-free subgroup of index $c / p$ or $2 c / p$. Hence $G$ has a torsion-free subgroup of index $2 c$, since one of index $c$ is ruled out by Lemma 4 .

Case 6. $m_{1}$ even, all the $m_{i}$ coprime in pairs.

Let $p, m$ be odd primes, $p>m$, dividing distinct odd $m_{i}$, and let $n=2$. Then $p, m, n$ satisfy the hypothesis of Lemma 3 , and hence as in Case 1 we can find a subgroup $H$ of $G$ such that $[G: H]=p$ and $H$ has a presentation (1) or (2) in which the $\mathrm{lcm}$ is $c / p$. By inductive hypothesis $H$ has a torsion-free subgroup of index $c / p$ or $2 c / p$. Hence $G$ has a torsion-free subgroup of index $2 c$ as required, since one of index $c$ is ruled out by Lemma 4 .

\section{REFERENCES}

1. T. C. Chau, A note concerning Fox's paper on Fenchel's conjecture, Proc. Amer. Math. Soc. 88 (1983), 584-586.

2. H. S. M. Coxeter and W. O. J. Moser, Generators and relations for discrete groups, Ergeb. Math. Grenzgeb., Bd. 14, 3rd ed., Springer-Verlag, Berlin and New York, 1972.

3. A. L. Edmonds, J. H. Ewing and R. S. Kulkarni, Torsion free subgroups of Fuchsian groups and tessellations of surfaces, Bull. Amer. Math. Soc. (N.S) 6 (1982), 456-458.

4. R. H. Fox, On Fenchel's conjecture about F-groups, Mat. Tidsskr. B. 1952 (1952), 61-65.

5. A. H. M. Hoare, A. Karrass and D. Solitar, Subgroups of finite index in Fuchsian groups, Math. Z. 120 (1971), 289-298.

6. Subgroups of infinite index in Fuchsian groups, Math. Z. 125 (1972), 59-69.

7. W. Magnus, A. Karrass and D. Solitar, Combinatorial group theory, 2nd rev. ed., Dover, New York, 1976.

Department of Mathematics, York University, Downsview, Toronto, Ontario, Canada M3J $1 \mathrm{P3}$ 